\title{
La transmisión digital de las agendas políticas en las Elecciones Locales de 2015 de la ciudad de Valencia
}

Political agendas transmission during the 2015 Municipal Elections of the city of Valencia

\author{
José Gamir-Ríos \\ Universitat de València \\ Orcid https://orcid.org/oooo-0oo1-5379-6573 \\ jose.gamir@uv.es
}

Resumen: El objetivo de la investigación es el estudio de la composición de las agendas temáticas partidistas en la campaña de las Elecciones Locales de 2015 en la ciudad de Valencia, así como de su transmisión en Internet a través de los sitios web de campaña, de las cuentas en Twitter de partidos y candidatos y de las piezas periodísticas publicadas en los cibermedios con cobertura informativa municipal. El trabajo constata que las formaciones construyeron sus agendas oficiales a partir, sobre todo, de asuntos relacionados con la política sectorial. Partidos y candidatos redefinieron sus agendas en Twitter y utilizaron la red de microblogging de manera instrumental, para la difusión de sus mensajes, el llamamiento al voto y la movilización. No consiguieron marcar la agenda de la comunidad de usuarios; tampoco se produjeron alineamientos claros con las temáticas tratadas por los principales cibermedios.

Palabras clave: Comunicación política; elecciones; redes sociales; prensa local; análisis de contenido

\begin{abstract}
The purpose of the investigation is to analyze the thematic composition of the political agendas for the 2015 Municipal Elections of the city of Valencia, as well as its transmission via Internet through Campaign Webpages, Political Parties' and Candidates' Twitter accounts and within journalistic pieces published in Online Media with Municipal coverage. This report finds that the political groupings mainly built their official agenda based on policy issues. Political Parties and Candidates redefined their agendas in Twitter and instrumentally used the microblogging network to spread their message, call people to vote and mobilize them. They weren't able to set the user community agenda; no apparent alignments with the themes covered by the main Online Media were made.
\end{abstract}

Keywords: Political communication, elections, social media, local press, content analysis

Fecha de recepción: 21/06/2020

Fecha de aprobación: 28/09/2020

Cómo citar este artículo: Gamir-Ríos, J. (2020). La transmisión digital de las agendas políticas en las Elecciones Locales de 2015 de la ciudad de Valencia. Revista de Comunicación Política, 2, 55-79. https://doi.org/10.29105/rcp2-3 


\section{Introducción}

La transmisión de las agendas temáticas y de sus atributos a través de los medios ha sido siempre uno de los principales objetivos de los partidos políticos en las campañas electorales. Sin embargo, la evolución a un ecosistema mediático híbrido, la consolidación de las redes sociales en el ámbito de la comunicación política, especialmente Twitter, y su uso instrumental por parte de partidos y candidatos han alterado los flujos habituales de la comunicación electoral. Por un lado, la actividad de los políticos en redes sociales contribuye a modular el contexto de decisión de los medios sobre los temas a tratar y los eventos a cubrir, incidiendo así en el proceso de agenda building. Por otro, los medios tradicionales han perdido su monopolio en el establecimiento de la agenda pública, de manera que constituyen solo una de las fuentes tomadas en consideración por las comunidades de ciudadanos en la fusión de las agendas de sus miembros. Todo ello hace conveniente el estudio conjunto de los mensajes emitidos por las formaciones, las piezas publicadas por los medios de comunicación y la agenda general de los usuarios de la red de microblogging para determinar su posible alineamiento temático.

Las elecciones al Ayuntamiento de Valencia celebradas en mayo de 2015 supusieron un ejemplo de la situación electoral española, tanto por las estimaciones demoscópicas previas como, por el planteamiento de las campañas digitales y por los resultados de los comicios. Resultan, por ello, un objeto de estudio conveniente para analizar los nuevos flujos de comunicación política en internet en periodo electoral.

\section{Las elecciones locales de 2015 en la ciudad de Valencia}

Dichos comicios ejemplifican las estimaciones porque, como en otros municipios, la mayoría de las encuestas pronosticaba una nueva victoria del Partido Popular (PP), que lideraba el gobierno municipal desde 1991 y que había logrado cinco mayorías absolutas consecutivas desde 1995. Sin embargo, también como en otras ciudades, los estudios cuantitativos estimaban la pérdida de la mayoría absoluta por parte de esta formación. Además, también como en el resto de España, las encuestas contemplaban un descenso del voto a las federaciones autonómicas del Partido Socialista Obrero Español (PSPV-PSOE) y de Izquierda Unida (EUPV), así como la obtención de representación por parte de Ciudadanos (C's) y de la candidatura municipalista en la que se integraba Podemos en la ciudad, València en Comú (VALC). Se preveía, por tanto, un escenario postelectoral abierto en el que la victoria por mayoría simple del PP podía resultarle insuficiente para seguir liderando el gobierno municipal, como consecuencia de un posible acuerdo postelectoral entre el PSPV y las candidaturas presentadas a la izquierda de su espacio político; en el caso de la ciudad, por Compromís -coalición estable integrada en el ámbito autonómico y local por un partido nacionalista, el Bloc Nacionalista Valencià, uno ecosocialista, Iniciativa pel Poble Valencià, y uno ecologista, Verds-Equo- y por la propia València en Comú. 
A su vez, ejemplifican el planteamiento de la campaña porque, como veremos, se consolidó en el ámbito municipal la utilización de Facebook y Twitter como herramientas fundamentales para la comunicación política online, iniciada en España en las Elecciones Generales de 2011 (Gamir Ríos, 2016) y reforzada en las Elecciones al Parlamento Europeo celebradas en mayo de 2014 (López García et al., 2015), y en las posteriores Elecciones Generales celebradas en diciembre de 2015 (Gamir Ríos et al., 2017).

Y, finalmente, ejemplifican también los resultados (PP, 10; Compromís, 9; C's, 6; PSPV; 5; VALC, 3; EUPV, o). Por un lado, se confirmó el tránsito del bipartidismo al multipartidismo, iniciado en las Elecciones al Parlamento Europeo celebradas en mayo de 2014 con la obtención de representación por parte de Podemos y Ciudadanos en la cámara comunitaria. Por otro, tras el descenso de votos del PSPV-PSOE, y como en Madrid, Barcelona, Zaragoza, A Coruña, Cádiz o Santiago de Compostela, entre otras capitales autonómicas o provinciales, el gobierno de la ciudad pasó a estar liderado por una formación perteneciente al espacio político situado a la izquierda del Partido Socialista, en este caso, por la coalición Compromís. En definitiva, los resultados profundizaron en la tendencia de "giro a la izquierda y hundimiento del bipartidismo" iniciada en España en las Elecciones al Parlamento Europeo de 2014 (Boix Palop \& López García, 2014).

\section{Twitter, agendas y medios en la comunicación política}

La creciente generalización de las redes sociales ha creado en la comunicación política una nueva esfera pública digital que "permite a la ciudadanía publicar información y opinión de manera instantánea y global” (Fenoll \& Cano Orón, 2019, p. 38). La emergencia de este nuevo espacio no solo ha consolidado la tendencia hacia la autocomunicación de masas (Castells, 2009), sino que también ha motivado la evolución de las campañas electorales desde el monopolio mediático previo hasta su actual ámbito de disputa digital (Dader, 2017).

El tradicional papel central de los medios convencionales - prensa, radio, televisión- en la comunicación política (Mazzoleni, 2010) se ha visto, así, superado. Ello es consecuencia, también, del surgimiento y de la consolidación de medios de comunicación digitales, que han derivado en la creación de un ecosistema híbrido (Chandwick, 2013) en el que los nuevos medios conviven con los preexistentes. Además de por la coexistencia de actores políticos dispares y por la multiplicación de medios, la nueva situación se caracteriza por la fragmentación de las audiencias y por el consumo transmedia (Jenkins, 2008). La recepción, que puede continuar iniciándose en los medios tradicionales, se extiende a través de los cibermedios y de las redes sociales, lo que diversifica las direcciones de los flujos comunicativos (López-García, 2016).

Los medios experimentan, de este modo, mayores dificultades para controlar la agenda pública, pues no solo compiten con los contenidos creados por los usuarios, sino también con el propio 
uso instrumental de la red por parte de partidos y candidatos. La incorporación de Twitter a la comunicación política, cuyas repercusiones en los procesos de agenda building han sido recopiladas ya en diversas ocasiones (ver, por ejemplo, Aruguete, 2017), ha supuesto un ajuste en las normas y prácticas profesionales de los periodistas a las normas y prácticas en evolución de dicha red de microblogging -especialmente entre los periodistas que trabajan para medios de comunicación locales o menos prestigiados- (Lasorsa et al., 2012). Los tuits políticos, además, contribuyen a modular tanto los eventos que cubren los periodistas, como las fuentes que entrevistan, las citas que usan y el contexto en el que se basan para decidir cómo cubrir un tema (Parmelee, 2013).

El modelo comunicativo de esta red de microblogging -breve, cuasi sincrónico, descentralizado, viralizable- la convierte "en un instrumento idóneo para que los líderes de opinión traten de marcar la agenda" (López García et al., 2015, p. 217). De hecho, su utilización se encuentra consolidada en el ámbito de la comunicación política pues, aunque las campañas no han abandonado las herramientas tradicionales, las combinan con los nuevos canales digitales para adaptarse al sistema híbrido anteriormente descrito (Casero-Ripollés, Feenstra \& Tormey, 2016). Sin embargo, lo hacen desde la unidireccionalidad (Giansante, 2015), intentando influir en los medios y movilizar a los simpatizantes (Kreiss, 2016), pero sin aprovechar su potencial dialógico (Alonso-Muñoz et al., 2016). En definitiva, los partidos emplean las redes sociales no solo para intensificar un tipo de comunicación similar a una red, sino sobre todo para la autorrepresentación, trasladando a los usuarios contenido creado por ellos mismos (Nitschke et al., 2014). Ello no deja de ser una adaptación a internet de la estrategia de control político de la información tradicionalmente denominada going public, consistente en evitar la mediación periodística y apelar de manera directa al electorado para solicitar su apoyo. La pretensión de dicha técnica es asegurar "que el mensaje político llega a los destinatarios en su integridad, sin la recontextualización y los cortes operados por las rutinas informativas y evitando la interpretación periodística” (Casero Ripollés, 2009, p. 360).

De esta forma, las redes sociales se han convertido en el nodo central de los flujos de comunicación política (Vaccari \& Valeriani, 2016); un espacio en el que, en el caso de Twitter, numerosas fuentes compiten por el establecimiento de la agenda (Jungherr, 2014). Los actores tradicionales de la comunicación política, mediáticos o partidistas, coexisten con muchos otros actores emergentes que, como la propia ciudadanía, tratan de influir en el espacio público digital desde un mismo nivel (Baviera, 2018). En este escenario de pérdida del monopolio del establecimiento de la agenda por parte de los medios convencionales, el concepto de agenda melding resulta especialmente conveniente para el estudio de la formación y la transmisión de agendas en el ámbito digital (Calvo \& Aruguete, 2018).

La teoría de la fusión de agendas (Shaw et al., 1999) contempla que las personas se agrupan de manera natural en comunidades cuyos miembros comparten su percepción de la realidad, y que en el seno de dichas comunidades reciben nuevas informaciones, las aceptan o rechazan y las propagan o no en función de la coherencia de dichos estímulos con la ideología y cosmovisión comunes, de 
manera que fusionan sus agendas con las del resto de miembros del grupo. Estas agrupaciones de ciudadanos-electores, en cuya conexión desempeñan un papel fundamental las tecnologías de la información y la comunicación, coexisten con dos modelos de comunicación: uno vertical y masivo, correspondiente a los medios tradicionales (prensa, radio y televisión) y otro horizontal y específico, correspondiente a los medios digitales, en consonancia con el actual ecosistema mediático híbrido. De este modo, las informaciones procedentes de ambos modelos penetran y se propagan en dichas comunidades en función de la coherencia que guarden con sus valores. En definitiva, la teoría sostiene que "distintos segmentos de votantes componen agendas de manera diferente utilizando diversas combinaciones de fuentes mediáticas” (Vargo et al., 2014, p. 15).

Las redes sociales presentan tres características que les otorgan relevancia en este proceso (López-López et al., 2020, p. 30): 1) "permiten la adquisición por parte de un actor político o de un tema de una variedad mucho más amplia de atributos que los medios de comunicación tradicionales"; 2) "construyen comunidades virtuales con ciudadanos que comparten comportamientos, gustos e ideología, por lo que tienen una imagen del mundo muy similar" y que, con su uso, ven satisfecha su necesidad de orientación; y 3) facilitan la propagación de atributos con una latencia menor a la de los medios tradicionales, siempre que no se produzca una disociación con los valores del grupo. No en vano, como señalan Calvo \& Aruguete (2018), “en estas comunidades, los usuarios son expuestos a contenidos -temas, figuras públicas y sus atributos- que se ajustan a sus preferencias y aumentan el placer informativo que deviene de compartir códigos comunes", lo que "estructura dinámicas de diseminación de información que incluyen los artículos publicados por medios tradicionales, no tradicionales y por otros usuarios" (p. 193).

Numerosos trabajos en el ámbito internacional, profusamente recopilados por Jungherr (2015), Vergeer (2015) y Campos-Domínguez (2017) en sendas revisiones, se han ocupado ya del estudio del papel de Twitter en la comunicación política. Muchos de ellos han investigado las estrategias y los mensajes desplegados por partidos y candidatos en el contexto de las campañas electorales. En España, los más recientemente realizados sobre campañas de ámbito estatal han tomado como objeto de estudio las Elecciones Generales de 2015 (López-García, 2016; Machado Flores \& Capdevilla Gómez, 2016; Cervi \& Roca, 2017; Gamir Ríos et al., 2017; Cervi \& Roca Trenchs, 2018) y las Elecciones Generales de 2016 (Casero-Ripolles, Miquel-Segarra \& Alonso-Muñoz, 2016; López-Meri et al., 2017; Alonso-Muñoz \& Casero-Ripollés, 2018; García-Ortega \& Zugasti Azagra, 2018).

\section{Objetivo y preguntas de investigación}

El objetivo de esta investigación es el estudio de la composición de las agendas temáticas en campaña electoral de las formaciones que concurrieron a las Elecciones Locales de 2015 en la ciudad de Valen- 
cia y obtuvieron representación (PP, Compromís, C's, PSPV-PSOE y VALC), así como de su transmisión en Internet a través de los sitios web de campaña, de las cuentas en Twitter de partidos y candidatos (Rita Barberá, Joan Ribó, Fernando Giner, Joan Calabuig y Jordi Peris, respectivamente) y de las piezas periodísticas publicadas en los principales cibermedios con cobertura informativa municipal.

Las cuatro preguntas que guían la investigación y que, en consecuencia, estructuran el corpus de trabajo en sendas secciones son:

PI1. ¿Cuál es la composición de las agendas temáticas de los textos difundidos por las candidaturas a través de sus sitios web?

PI2. ¿Cuál es la composición de las agendas temáticas de las cuentas de los partidos y los candidatos en Twitter, y en qué medida se relacionan con las anteriores?

PI3. ¿En qué medida se insertan los mensajes emitidos en Twitter por las cuentas de partidos y candidatos en la agenda temática de la comunidad de usuarios de esta red?

PI4. ¿Cuál es la composición de las agendas temáticas de los cibermedios y en qué medida están alineadas con las agendas partidistas?

\section{Metodología}

La investigación se circunscribe a un marco temporal que abarca el periodo oficial de la campaña electoral, del 8 al 22 de mayo de 2015, y que incluye también la jornada de reflexión, la fecha de la votación y el día posterior a los comicios. La metodología principal es el análisis de contenido (Krippendorff, 1990) y contempla la codificación del tema de los mensajes como variable única y común a la totalidad de la muestra.

\section{Muestra}

Para dar respuesta a las preguntas de investigación, el trabajo examina una muestra formada por 4.463 unidades de análisis y estructurada en cuatro secciones.

\section{Agendas temáticas de los sitios web de campaña}

La sección de la muestra referida a la PI1 está compuesta por los textos difundidos en las secciones de actualidad de las páginas webs de la candidata del Partido Popular, Rita Barberá (15); de Compromís per València (20); de Ciudadanos-C's de la ciudad de Valencia y su candidato, Fernando 
Giner (43); del PSPV-PSOE Valencia y su candidato, Joan Calabuig (29); y de València en Comú y su candidato, Jordi Peris (44). En el caso del PP, se ha determinado la agenda exclusivamente a partir de los textos publicados en la página web de su candidata pues la formación no dispuso de página web propia. En el caso de Compromís se ha recurrido solo a la página web del partido, pues la de su candidato, Joan Ribó, no se actualizó durante la campaña. Las piezas analizadas fueron recopiladas a diario de manera manual en la franja horaria comprendida entre las 23:00 y las 24:00.

\section{Agendas temáticas de partidos y candidatos en Twitter}

Por su parte, la sección correspondiente a la PI2 está formada por los mensajes de contenido propio publicados en Twitter por las cuentas @ppvlc (o), @ ritaalcaldesa (159), @ compromisvlc (607), @joanribo (90), @csvalencia_c (288), @fginer (48), @pspv_psoevlc (426), @joancalabuig (116), @vlcencomu (484) y @jordiperis2015 (666). Para facilitar el seguimiento cualitativo de la campaña, las cuentas analizadas fueron objeto de una monitorización diaria en dos franjas horarias, entre las 14:00 y las 15:00 y entre las 23:00 y las 24:00; sin embargo, por razones de operatividad, la muestra fue exportada en su totalidad entre las 00:00 y las 01:00 del 26 de mayo mediante la herramienta Twittonomy. Pese a que la actividad en Twitter de las cuentas que componen la muestra ascendió a 7.208 tuits, el corpus final al que se ha aplicado el análisis de contenido está integrado solo por los 2.884 mensajes emitidos de contenido propio, habiéndose descartado los retuits y las respuestas. La muestra incluye las cuentas tanto de los partidos como de los candidatos, excepto en el caso del Partido Popular, cuya agrupación de la ciudad de Valencia no realizó ninguna publicación de contenido propio.

\section{Penetración de las agendas temáticas en Twitter}

A su vez, la sección vinculada a la PI3 está integrada por los 529 hashtags que alcanzaron la condición de trending topic en Twitter en el área de Valencia, de acuerdo con los datos recopilados a través de la herramienta online Trendinalia, completados con los ofrecidos por el sitio web Trendsmap.

\section{Agendas temáticas de los cibermedios}

Por último, la sección relativa a la PI4 está constituida por los 899 textos sobre la campaña electoral municipal o sobre la actividad del Ayuntamiento de Valencia aparecidas en las ediciones digitales de El País (47), El Mundo (85), Levante-EMV (287) y Las Provincias (305), así como en los cibermedios nativos El Diario (66) y Valencia Plaza (109), con independencia de su género periodístico o de la sección en la que se publicaron. El País y El Mundo eran los dos cibermedios generalistas 
más leídos en España con secciones dedicadas a la Comunidad Valenciana: 14.832.000 y 13.804.000 usuarios únicos en mayo de 2015, respectivamente, según los datos de comScore, MMX Multiplataform. Levante-EMV y Las Provincias eran los dos cibermedios editados en la provincia de Valencia cuyos referentes impresos acumulaban mayor audiencia: 259.000 y 128.000 lectores, respectivamente, según los datos de la primera oleada de 2015 del Estudio General de Medios, correspondiente al periodo abril de 2014-marzo de 2015. El Diario era entonces el segundo cibermedio nativo más leído de España, con una audiencia acumulada de 4.860.00o usuarios únicos en el mes de mayo de 2015 según los datos de comScore, MMX Multiplataform, superado solo por El Confidencial, no incluido en la muestra por la escasa cobertura informativa que dedicaba a la actualidad valenciana. Por último, Valencia Plaza era el cibermedio nativo digital de referencia entre los editados en la provincia de Valencia, con una audiencia de 566.162 lectores únicos en el mes de mayo de 2015, según los datos de OJD interactiva. Las páginas analizadas fueron objeto de un seguimiento diario en la franja horaria comprendida entre las 23:00 y las 01:00, tanto para monitorizar su actividad como, sobre todo, para recopilar de manera manual los materiales que posteriormente iban a ser objeto del análisis de contenido.

\section{Método y operacionalización}

La variable común en el análisis de las cuatro secciones del corpus es la adscripción temática de los mensajes analizados (v1), siguiendo la técnica de análisis categorial temático de contenido (Bardin, 1986, p. 119). Para ello, hemos distinguido tres niveles de categorías que estructuran la clasificación de la totalidad de la muestra. En un primer nivel, hemos manejado las cuatro macrocategorías propuestas por Patterson. Según las definiciones de Mazzoleni (2010): a) political issues, "temas concernientes a la esfera más abstracta de la confrontación político-electoral”; b) policy issues, "cuestiones relativas a las políticas gubernamentales, administrativas y legislativas, pero también a los 'problemas concretos"; c) personal issues, "categoría que entra en la dimensión personal e individual de los sujetos políticos"; y d) campaign issues, que "abarcan aspectos muy variados relativos a las estrategias y a la organización de las campañas electorales” (pp. 210-213). En un tercer nivel, hemos desglosado los policy issues en 52 categorías, tras analizar, por un lado, las competencias municipales establecidas en la Ley 7/1985, de 2 de abril, Reguladora de las Bases del Régimen Local; por otro, el organigrama del Ayuntamiento de Valencia a fecha de inicio de la campaña electoral; y, por último, los temas presentes en la agenda mediática y política durante los meses previos a la campaña electoral. En un segundo nivel, hemos agrupado dichos 52 temas en 7 bloques mayores, relacionados con las áreas más frecuentes de la gestión municipal o del discurso político o mediático local. El proceso de codificación ha servido para registrar un mínimo de un tema y un máximo de tres por cada unidad de análisis, a excepción de los hashtags que integran la sección de la muestra referida a la PI3, en los que, por su brevedad y sentido unívoco, se ha registrado solo uno. 
Tabla 1. Categorías temáticas de codificación

\begin{tabular}{ll}
\hline Primer nivel & Segundo nivel \\
\hline Political issues & Hacienda e inversiones \\
& Buen gobierno \\
& Economía y empleo \\
& Urbanismo y espacios naturales \\
& Movilidad, espacio público y calidad ambiental \\
Policy issues & Seguridad ciudadana \\
& Política social \\
& Educación, cultura y deporte \\
& Otros
\end{tabular}

Campaign issues

Personal issues

La codificación fue realizada por un único investigador en los meses de junio y julio de 2015, con carácter inmediatamente posterior a la finalización de la campaña electoral para facilitar la incorporación al análisis de contenido del criterio formado gracias al seguimiento diario del proceso. Posteriormente, en agosto de 2020 y con motivo de la redacción del presente trabajo, el mismo investigador realizó una prueba de fiabilidad sobre el 10\% de cada sección de la muestra para asegurar la estabilidad de la investigación. El coeficiente Alfa de Krippendorff $(\alpha=.82$, en el caso de la sección del corpus referida a la PI1; $\alpha=.79$, en la PI2; $\alpha=.87$, en la PI3; y $\alpha=.80$, en la PI4) señala una adecuada fiabilidad (Igartua, 2006), lo que permite validar los resultados.

El análisis cuantitativo de contenido se ha completado con una aproximación cualitativa a las secciones de la muestra correspondientes a la PI1, PI2 y PI4 mediante el análisis del discurso de los mensajes. Dicho análisis se ha centrado en la observación de dos variables. Por un lado, la coherencia de los discursos y las agendas de partidos y candidatos con las estrategias de las formaciones, determinadas sobre todo por aspectos como su presencia o ausencia de la corporación 2011-2015, su posición en dicha corporación -gobierno u oposición-, su previsión de resultados, su adscripción ideológica respecto al eje izquierda-derecha y su naturaleza emergente o consolidada. Por otro, la posible asimilación de dichas agendas por parte de los cibermedios, así como la coherencia de los discursos mediáticos con las adscripciones ideológicas de sus líneas editoriales. 


\section{Resultados}

\section{Las agendas temáticas de los sitios web de campaña}

Respecto a la PI1, ¿̇cuál es la composición de las agendas temáticas de los textos difundidos por las candidaturas a través de sus sitios web de campaña?, todos los partidos priorizaron en sus páginas los asuntos relacionados con la política sectorial excepto Ciudadanos. Como muestra la Tabla 2, Compromís fue la formación en cuya agenda más estuvo presente dicho bloque, seguida del PPCV y del PSPV-PSOE; la presencia relativa agregada de dichos temas fue en los tres casos equivalente o superior a la mitad. València en Comú y Ciudadanos estuvieron, en cambio, por debajo de dicho porcentaje.

Tabla 2. Composición de las agendas partidistas oficiales por bloques temáticos

\begin{tabular}{lccccc}
\hline Bloque & PPCV & Compromís & C's & PSPV & VALC \\
\hline Political issues & $22.00 \%$ & $4.65 \%$ & $22.81 \%$ & $11.11 \%$ & $13.83 \%$ \\
Policy issues & $52.00 \%$ & $76.74 \%$ & $12.28 \%$ & $50 \%$ & $46.81 \%$ \\
Campaign issues & $22.00 \%$ & $13.95 \%$ & $52.63 \%$ & $31.94 \%$ & $36.17 \%$ \\
Personal issues & $4.00 \%$ & $4.65 \%$ & $12.28 \%$ & $6.94 \%$ & $3.19 \%$ \\
\hline
\end{tabular}

A su vez, dentro del bloque de política sectorial, tal y como se constata en la Tabla 3, en el partido de centroderecha entonces gobernante, el Partido Popular centró su agenda, por este orden, en los asuntos relacionados con la economía, el buen gobierno, la cultura festiva e identitaria y la hacienda municipal. Su estrategia discursiva combinó elementos de recuperación económica y creación de empleo con la inversión realizada en los barrios de la ciudad, la defensa de las que la formación identifica como señas de identidad valencianas y la congelación de impuestos.

Las prioridades sectoriales difirieron en el centroizquierda valencianista y ecosocialista de Compromís, el centroizquierda socialdemócrata/socioliberal del PSPV-PSOE, y la izquierda transformadora postcomunista de València en Comú. El área de buen gobierno encontró un lugar destacado en los tres casos; principal en los dos primeros y secundario pero relevante en el tercero. El discurso al respecto de los tres partidos coincidió en presentar a Barberá y al Partido Popular como un equipo de gobierno corrupto, poco transparente y que realizaba un uso partidista de los fondos públicos. Al margen de ello, dentro de la misma área, los partidos de izquierdas consolidados -Compromís y el PSPV-PSOE- se ocuparon más de la descentralización de la inversión que el emergente -València en Comú-, al hablar de las desigualdades entre barrios y al hacer propuestas concretas por territorios. Además, los partidos ideológicamente situados más a la izquierda -Compromís y València en Comú- 
se ocuparon más de la participación ciudadana que el más cercano al centro político -PSPV-PSOE-, al proponer medidas específicas de empoderamiento ciudadano.

Tabla 3. Composición de las agendas partidistas oficiales por áreas sectoriales

\begin{tabular}{lccccc}
\hline Policy issues & PPCV & Compromís & C's & PSPV & VALC \\
\hline Hacienda e inversiones & $6 \%$ & $0 \%$ & $1.75 \%$ & $0 \%$ & $1.06 \%$ \\
Buen gobierno & $12 \%$ & $30.23 \%$ & $3.51 \%$ & $18.06 \%$ & $9.57 \%$ \\
Economía y empleo & $18 \%$ & $11.63 \%$ & $0 \%$ & $8.33 \%$ & $11.70 \%$ \\
$\begin{array}{l}\text { Urbanismo y espacios natura- } \\
\text { les }\end{array}$ & $2.00 \%$ & $13.95 \%$ & $1.75 \%$ & $4.17 \%$ & $5.32 \%$ \\
$\begin{array}{l}\text { Mov., esp. público y c. ambien- } \\
\text { tal }\end{array}$ & $0 \%$ & $9.30 \%$ & $0 \%$ & $0 \%$ & $1.06 \%$ \\
Seguridad ciudadana & $0 \%$ & $0 \%$ & $0 \%$ & $0 \%$ & $0 \%$ \\
Política social & $6 \%$ & $0 \%$ & $1.75 \%$ & $13.89 \%$ & $11.70 \%$ \\
Educación, cultura y deporte & $8 \%$ & $6.98 \%$ & $3.51 \%$ & $4.17 \%$ & $3.19 \%$ \\
Otros & $0 \%$ & $4.65 \%$ & $0 \%$ & $1.39 \%$ & $3.19 \%$ \\
\hline
\end{tabular}

Las agendas oficiales de los tres partidos coincidieron con la del Partido Popular en otorgar un papel relevante al área de economía y empleo. Sin embargo, mientras el PP exhibió los logros de su trayectoria, Compromís, PSPV-PSOE y València en Comú criticaron las desigualdades que atribuían a su modelo de crecimiento y propugnaron alternativas basadas en el empleo de calidad y el comercio de proximidad (los tres casos), en el emprendimiento (PSPV-PSOE) o el cooperativismo (València en Comú), y en una redefinición de las políticas turísticas hacia la desestacionalización y la descentralización (Compromís y PSPV-PSOE). Al margen de ello, las agendas del PSPV-PSOE y de València en Comú otorgaron una atención igualmente relevante a las políticas sociales, en especial a la lucha contra la exclusión social, mientras que Compromís completó su agenda con asuntos relacionados con el urbanismo y la movilidad.

Las formaciones emergentes -Ciudadanos y València en Comú- y la consolidada gobernante -Partido Popular- fueron las opciones que más presencia otorgaron en sus agendas al bloque de política general a través de los denominados political issues. En los tres casos, a través de cuestiones relativas a un eje que València en Comú formuló de acuerdo con la lógica vieja-nueva política, que Ciudadanos presentó como continuidad-cambio y que el Partido Popular trató de reformular como estabilidad-incertidumbre y, sobre todo, como moderación-radicalidad. Asimismo, los dos partidos con experiencia de gobierno municipal recordaron los logros de sus mandatos: el PP, en el reciente periodo 1991-2015, y el PSPV-PSOE, en el lejano 1979-1991. A su vez, el emergente de más reciente 
creación, València en Comú, recordó con frecuencia su condición de candidatura referente de Podemos en la ciudad.

El bloque relativo a la campaña electoral fue el segundo con mayor presencia en las agendas de todos los partidos -excepto en la de Ciudadanos, en la que ocupa el primer lugar-. Sin embargo, fueron las formaciones emergentes las que más esfuerzos dedicaron en dicho bloque a la difusión de los temas relacionados con sus eventos y actos públicos. El Partido Popular, como candidatura en el gobierno municipal, fue el que más espacio dedicó a las críticas de las campañas de sus adversarios.

\section{Las agendas temáticas de partidos y candidatos en Twitter}

En cuanto a la PI2, ¿̇cuál es la composición de las agendas temáticas difundidas por las cuentas de los partidos en Twitter, y en qué medida se relacionan con las transmitidas a través de los sitios web de campaña?, todos los partidos priorizaron los campaign issues en las agendas de sus cuentas en la red de microblogging. València en Comú fue la formación en cuya agenda más estuvo presente dicho bloque, seguida de Compromís, Ciudadanos y el PSPV-PSOE. Sin embargo, el peso en la agenda temática fue diferente: superior al 60\% en el caso de la candidatura municipal vinculada a Podemos, en torno al 50\% en los casos de la coalición valencianista y de la candidatura liderada por Fernando Giner, y alrededor del 45\% en el caso de la formación socialista. En todos los casos, los asuntos de campaña más presentes fueron los relacionados con la realización de llamamientos al voto y con la difusión de actos públicos. Las diferencias entre las agendas partidistas radicaron, por tanto, en la importancia otorgada al resto de asuntos.

Tabla 4. Composición de las agendas partidistas en Twitter por bloques temáticos

\begin{tabular}{lccccc}
\hline Bloque & PPCV & Compromís & C's & PSPV & VALC \\
\hline Political issues & O\% & $9.57 \%$ & $24.87 \%$ & $12.92 \%$ & $12.26 \%$ \\
Policy issues & o\% & $28.87 \%$ & $16.64 \%$ & $34.61 \%$ & $21.34 \%$ \\
Campaign issues & o\% & $51.34 \%$ & $51.26 \%$ & $45.13 \%$ & $62.11 \%$ \\
Personal issues & o\% & $10.22 \%$ & $7.06 \%$ & $7.34 \%$ & $4.29 \%$ \\
\hline
\end{tabular}

A su vez, como se muestra en la Tabla 4, el segundo bloque con mayor presencia en las agendas de las cuentas en Twitter de Compromís, del PSPV-PSOE y de València en Comú fue el correspondiente a la política sectorial; mientras que en el caso de Ciudadanos fue el vinculado a la política general. A su vez, dentro del bloque de policy issues, como se evidencia en la Tabla 5, el área más presente en todos los casos fue la referida al buen gobierno, con alusiones al despilfarro, la corrupción, 
la transparencia y el uso partidista de los fondos públicos. Solo la agenda del PSPV-PSOE contempló una presencia superior al 5\% de alguna otra área: política social y economía y empleo.

Tabla 5. Composición de las agendas partidistas en Twitter por áreas sectoriales

\begin{tabular}{|c|c|c|c|c|c|}
\hline Policy issues & $\overline{\mathrm{PPCV}}$ & Compromís & $\bar{C}$ C's & PSPV & VALC \\
\hline Hacienda e inversiones & O\% & $1.05 \%$ & $1.01 \%$ & $0.33 \%$ & $0.20 \%$ \\
\hline Buen gobierno & $0 \%$ & $12.33 \%$ & $4.54 \%$ & $10.19 \%$ & $10.27 \%$ \\
\hline Economía y empleo & $0 \%$ & $2.60 \%$ & $3.87 \%$ & $7.45 \%$ & $1.10 \%$ \\
\hline $\begin{array}{l}\text { Urbanismo y espacios } \\
\text { naturales }\end{array}$ & $0 \%$ & $2.92 \%$ & $1.34 \%$ & $4.05 \%$ & $0.80 \%$ \\
\hline $\begin{array}{l}\text { Mov., esp. público y c. } \\
\text { ambiental }\end{array}$ & $0 \%$ & $3.08 \%$ & $2.18 \%$ & $0.99 \%$ & $2.89 \%$ \\
\hline Seguridad ciudadana & $0 \%$ & $0.24 \%$ & $0 \%$ & $0 \%$ & $0 \%$ \\
\hline Política social & $0 \%$ & $2.60 \%$ & $1.85 \%$ & $7 \cdot 34 \%$ & $3.89 \%$ \\
\hline Educación, cultura y deporte & $0 \%$ & $3.24 \%$ & $1.85 \%$ & $3.61 \%$ & $1.60 \%$ \\
\hline Otros & $0 \%$ & $0.81 \%$ & $0 \%$ & $0.66 \%$ & $0.60 \%$ \\
\hline
\end{tabular}

Así, las formaciones de izquierda y centro-izquierda priorizaron las críticas o propuestas sectoriales, correspondientes a áreas concretas de la gestión municipal, mientras que Ciudadanos optó por aspectos más generales, relacionados sobre todo con el eje continuidad-cambio. El bloque de political issues ocupó el tercer lugar en las agendas del PSPV-PSOE y de València en Comú; en ambos casos a partir del mismo eje, que la candidatura socialista enfoca, como Ciudadanos, en torno a los polos continuidad-cambio, y que la formación municipalista desarrolla a partir de los extremos viejanueva política.

De tal modo, en relación con el segundo interrogante planteado en la PI2, las formaciones políticas, a excepción de Ciudadanos, no utilizaron su presencia en la red de microblogging para transmitir fielmente sus agendas oficiales. Así, las agendas temáticas de las cuentas en Twitter de Compromís, el PSPV-PSOE y València en Comú redujeron notablemente los asuntos relacionados con la política sectorial en beneficio de los referidos a la campaña electoral, sobre todo a la realización de llamamientos al voto y a la difusión de eventos y actos públicos. En concreto, la presencia de los policy issues experimentó un descenso de dos tercios en la agenda de la coalición valencianista, de un tercio en la de la candidatura socialista y de la mitad en la de la formación municipalista. Además, la importancia de las diferentes áreas sectoriales que conforman dicho bloque se vio alterada en todos los casos. 
Continuando con la respuesta a la PI2, los cinco candidatos otorgaron un papel principal a los asuntos correspondientes al bloque de campaign issues en la composición de las agendas temáticas de sus cuentas oficiales en Twitter, como también sucedió en las cuentas corporativas de la red de microblogging. Giner fue el candidato en cuya agenda más estuvo presente dicho bloque, seguido de Ribó, Barberá, Peris y Calabuig. Sin embargo, el peso en la agenda temática fue diferente, como se observa en la Tabla 6: superior al 50\% en los casos de los candidatos de Ciudadanos y de Compromís; en torno al $40 \%$ en los casos de los candidatos del PPCV, València en Comú y el PSPV-PSOE. En todos los casos, los asuntos de campaña más presentes en los mensajes publicados en las cuentas de los candidatos en Twitter son los relacionados con la realización de llamamientos al voto y con la difusión de los eventos y actos públicos. Las diferencias entre las agendas partidistas radicaron, de nuevo, en la importancia otorgada al resto de asuntos.

Tabla 6. Composición de las agendas de los candidatos en Twitter por bloques temáticos

\begin{tabular}{lccccc}
\hline Bloque & Barberá & Ribó & Giner & Calabuig & Peris \\
\hline Political issues & $23.51 \%$ & $18.05 \%$ & $11.34 \%$ & $16.59 \%$ & $25.98 \%$ \\
Policy issues & $15.76 \%$ & $21.05 \%$ & $16.49 \%$ & $34.93 \%$ & $21.82 \%$ \\
Campaign issues & $43.67 \%$ & $54.89 \%$ & $56.70 \%$ & $39.30 \%$ & $40.07 \%$ \\
Personal issues & $17.05 \%$ & $6.02 \%$ & $14.43 \%$ & $8.30 \%$ & $12.01 \%$ \\
\hline
\end{tabular}

El segundo bloque con mayor presencia en las agendas de las cuentas en Twitter de Ribó, Giner y Calabuig fue el correspondiente a la política sectorial, mientras que en las cuentas de Barberá y Peris fue el vinculado a la política general. El tercer bloque más presente en las agendas de Ribó y Calabuig fue el relacionado con la política general; en las de Barberá y Giner, el referido a los asuntos personales; en la de Peris, el de política sectorial. Por último, el bloque con menor presencia en las agendas de Ribó, Calabuig y Peris fue el vinculado a los personal issues; en la de Barberá, con los policy issues; y en la de Giner, con los political issues. La candidata del PPCV relegó así al último lugar de su agenda en Twitter la defensa de su gestión y las propuestas concretas de su programa, mientras que el de Ciudadanos lo hizo con unos temas, los relacionados con la política general, que sin embargo ocuparon un lugar destacado en la agenda oficial de su formación.

A su vez, como se observa en la Tabla 7, el bloque de policy issues experimentó una dispersión generalizada en función de los candidatos. Barberá priorizó el urbanismo y la economía y el empleo; Ribó, el buen gobierno y la economía y el empleo; Giner, la economía y el empleo y la educación; Calabuig, el buen gobierno, la política social y la educación; y Peris, el buen gobierno y la política social. Solo las agendas de Ribó y Calabuig contemplaron una presencia superior al $5 \%$ de algún área no principal. 
Tabla 7. Composición de las agendas de los candidatos en Twitter por áreas sectoriales

\begin{tabular}{lccccc}
\hline Policy issues & Barberá & Ribó & Giner & Calabuig & Peris \\
\hline Hacienda e inversiones & $2.58 \%$ & $0 \%$ & $0 \%$ & $0 \%$ & $0 \%$ \\
Buen gobierno & $2.33 \%$ & $9.77 \%$ & $3.09 \%$ & $12.23 \%$ & $13.05 \%$ \\
Economía y empleo & $3.36 \%$ & $6.77 \%$ & $5.15 \%$ & $3.93 \%$ & $1.50 \%$ \\
$\begin{array}{l}\text { Urbanismo y espacios } \\
\text { naturales }\end{array}$ & $3.62 \%$ & $2.26 \%$ & $0 \%$ & $3.49 \%$ & $1.15 \%$ \\
$\begin{array}{l}\text { Mov., esp. público y c. } \\
\text { ambiental }\end{array}$ & $0 \%$ & $0 \%$ & $2.06 \%$ & $1.75 \%$ & $0.46 \%$ \\
Seguridad ciudadana & $0 \%$ & $0 \%$ & $0 \%$ & $0 \%$ & $0 \%$ \\
Política social & $2.84 \%$ & $1.50 \%$ & $1.03 \%$ & $6.55 \%$ & $3.81 \%$ \\
Educación, cultura y deporte & $1.03 \%$ & $0.75 \%$ & $5.15 \%$ & $6.55 \%$ & $1.73 \%$ \\
Otros & $0 \%$ & $0 \%$ & $0 \%$ & $0.44 \%$ & $0.12 \%$ \\
\hline
\end{tabular}

Así, todos los candidatos otorgaron mayor importancia a la difusión de las actividades de campaña y al llamamiento al voto de sus posibles electores, en perjuicio de las críticas y propuestas sobre áreas de gestión concretas. Además, con relación al segundo enunciado de la PI2, todos ellos redefinieron en sus páginas de la red social la jerarquía de las diferentes áreas de gestión, con lo que la composición del bloque de política sectorial fue en todos los casos diferente a la registrada en las agendas oficiales.

\section{La penetración de las agendas temáticas en Twitter}

En relación con la PI3, que planteaba ċen qué medida se insertan los mensajes emitidos en Twitter por las cuentas de partidos y candidatos en la agenda temática general de la comunidad de usuarios de esta red de microblogging en el área de Valencia?, cabe destacar un alineamiento claramente minoritario. Los usuarios de Twitter convirtieron en trending topic durante el periodo analizado 1529 palabras, frases o cuentas en el área de Valencia. De ellas, 529 utilizaron el formato hashtag. Sin embargo, solo 168 usos de estas etiquetas -el 31.75\%- guardaron relación con la actualidad política, mientras que 361 -el $68.25 \%$ - no correspondieron a dicho ámbito.

A su vez, 123 de dichos usos de hashtags se refirieron a asuntos relacionados con la campaña electoral de ámbito nacional, autonómico o local, mientras que 45 aludieron a otros aspectos políticos. Los 123 etiquetados comprendieron 102 hashtags únicos que, como se observa en la Tabla 8, se ocuparon mayoritariamente de asuntos relacionados con la campaña electoral, bloque que registró una presencia del $54.9 \%$, mientras que los temas relativos a la política general ocuparon un lugar secun- 
dario, con una presencia del $\mathbf{1 8 . 6 3 \%}$. Los aspectos correspondientes a la política sectorial y a la dimensión personal de los candidatos recibieron una atención residual, con frecuencias relativas agregadas del $4.9 \%$ y del $1.96 \%$, respectivamente.

Tabla 8. Composición por bloques temáticos de la agenda de la comunidad de usuarios de Twitter a través de los hashtags convertidos en trending topics

\begin{tabular}{lcc}
\hline Bloque & Frecuencia absoluta & Frecuencia relativa \\
\hline Political issues & 19 & $18.63 \%$ \\
Policy issues & 5 & $4.90 \%$ \\
Campaign issues & 56 & $54.90 \%$ \\
Personal issues & 2 & $1.96 \%$ \\
Otros & 20 & $19.61 \%$ \\
\hline
\end{tabular}

La agenda política de los usuarios de Twitter difirió así de forma notable de las desarrolladas por las cuentas oficiales de los partidos y de los candidatos. En el primer caso, como se constata en la Tabla 9, las variaciones fueron de los 70.17 puntos porcentuales del PSPV-PSOE, la candidatura cuya agenda partidista en Twitter registró una desviación más acusada respecto a la de la comunidad de usuarios, a los 46.16 puntos de Ciudadanos, la candidatura cuya agenda más se aproximó a ella.

Tabla 9. Variación de la composición por bloques temáticos de las agendas partidistas en Twitter respecto a la comunidad de usuarios

\begin{tabular}{lcccccc}
\hline Bloque & $\begin{array}{c}\text { Frecuencia } \\
\text { relativa } \\
\text { Twitter }\end{array}$ & $\begin{array}{c}\text { Variación } \\
\text { relativa PP }\end{array}$ & $\begin{array}{c}\text { Variación } \\
\text { relativa } \\
\text { Compromís }\end{array}$ & $\begin{array}{c}\text { Variación re- } \\
\text { lativa Ciuda- } \\
\text { danos }\end{array}$ & $\begin{array}{c}\text { Variación re- } \\
\text { lativa PSPV }\end{array}$ & $\begin{array}{c}\text { Variación rela- } \\
\text { tiva VALC }\end{array}$ \\
\hline Political & $18.63 \%$ & - & $-9.06 \%$ & $6.25 \%$ & $-5.70 \%$ & $-6.36 \%$ \\
Policy & $4.90 \%$ & - & $23.97 \%$ & $11.74 \%$ & $29.71 \%$ & $16.43 \%$ \\
Campaign & $54.90 \%$ & - & $-3.56 \%$ & $-3.64 \%$ & $-9.78 \%$ & $7.21 \%$ \\
Personal & $1.96 \%$ & - & $8.26 \%$ & $5.10 \%$ & $5.38 \%$ & $2.33 \%$ \\
Otros & $19.61 \%$ & - & $-19.61 \%$ & $-19.44 \%$ & $-19.61 \%$ & $-19.61 \%$ \\
Desviación agregada & - & $64.46 \%$ & $46.16 \%$ & $70.17 \%$ & $51.94 \%$ \\
\hline
\end{tabular}


En el segundo, como se observa en la Tabla 10, las variaciones oscilaron entre los 72.74 puntos porcentuales de Calabuig, el candidato cuya agenda partidista en Twitter registró una desviación más acusada respecto a la de la comunidad de usuarios, y los 40.41 puntos de Ribó, el candidato cuya agenda más se aproximó a ella.

Tabla 10. Variación de la composición por bloques temáticos de las agendas de los candidatos en Twitter respecto a la comunidad de usuarios

\begin{tabular}{lcccccc}
\hline Bloque & $\begin{array}{c}\text { Frecuencia re- } \\
\text { lativa Twitter }\end{array}$ & $\begin{array}{c}\text { Variación } \\
\text { relativa } \\
\text { Barberá }\end{array}$ & $\begin{array}{c}\text { Variación } \\
\text { relativa } \\
\text { Ribó }\end{array}$ & $\begin{array}{c}\text { Variación } \\
\text { relativa Gi- } \\
\text { ner }\end{array}$ & $\begin{array}{c}\text { Variación } \\
\text { relativa Ca- } \\
\text { labuig }\end{array}$ & $\begin{array}{c}\text { Variación } \\
\text { relativa Pe- } \\
\text { ris }\end{array}$ \\
\hline Political & $18.63 \%$ & $4.89 \%$ & $-0.58 \%$ & $-7.29 \%$ & $-2.03 \%$ & $7.35 \%$ \\
Policy & $4.90 \%$ & $10.86 \%$ & $16.15 \%$ & $11.59 \%$ & $30.03 \%$ & $16.92 \%$ \\
Campaign & $54.90 \%$ & $-11.23 \%$ & $-0.01 \%$ & $1.80 \%$ & $-15.60 \%$ & $-14.83 \%$ \\
Personal & $1.96 \%$ & $15.09 \%$ & $4.05 \%$ & $12.47 \%$ & $6.34 \%$ & $10.05 \%$ \\
Otros & $19.61 \%$ & $-19.61 \%$ & $-19.61 \%$ & $-18.58 \%$ & $-18.73 \%$ & $-19.49 \%$ \\
\multicolumn{2}{l}{ Desviación agregada } & $61.68 \%$ & $40.41 \%$ & $51.73 \%$ & $72.74 \%$ & $68.65 \%$ \\
\hline
\end{tabular}

Como se observa en la Tabla 11, los mensajes etiquetados emitidos por Compromís registraron un grado de inserción en la agenda política de la comunidad de usuarios de Twitter en el área de Valencia durante el periodo analizado del 13.72\%; los de València en Comú, del 11.76\%; los del PSPVPSOE, del 8.82\%; y los de Ciudadanos, del 7.84\%. A su vez, como se evidencia en la Tabla 12, los mensajes etiquetados por Peris registraron un grado de inserción del 17.64\%; los de Ribó, del 4.90\%; los de Barberá y Calabuig, del 3.92\%; y los de Giner, del 0.98\%.

Tabla 11. Inserción de los hashtags utilizados por los partidos en la agenda temática de los usuarios de Twitter

\begin{tabular}{lcccccc}
\hline & $\begin{array}{c}\text { Usos de } \\
\text { hashtags }\end{array}$ & $\begin{array}{c}\text { Hashtags } \\
\text { únicos }\end{array}$ & $\begin{array}{c}\text { Frecuencia } \\
\text { media de } \\
\text { repetición }\end{array}$ & $\begin{array}{c}\text { TT entre los } \\
\text { hashtags } \\
\text { únicos }\end{array}$ & $\begin{array}{c}\text { Hashtags } \\
\text { propios entre } \\
\text { los TT }\end{array}$ & $\begin{array}{c}\text { Inserción } \\
\text { en agenda } \\
\text { de TT }\end{array}$ \\
\hline PP & - & - & - & - & - & - \\
Compromís & 512 & 84 & 6.10 & 14 & 4 & $13.72 \%$ \\
Ciudadanos & 140 & 24 & 5.83 & 8 & 6 & $7.84 \%$ \\
PSPV & 972 & 110 & 8.84 & 9 & 7 & $8.82 \%$ \\
VALC & 390 & 32 & 12.19 & 12 & 3 & $11.76 \%$ \\
\hline
\end{tabular}


Tabla 12. Inserción de los hashtags utilizados por los candidatos en la agenda temática de los usuarios de Twitter

\begin{tabular}{lcccccc}
\hline & $\begin{array}{c}\text { Usos de } \\
\text { hashtags }\end{array}$ & $\begin{array}{c}\text { Hashtags } \\
\text { únicos }\end{array}$ & $\begin{array}{c}\text { Frecuencia } \\
\text { media de } \\
\text { repetición }\end{array}$ & $\begin{array}{c}\text { TT entre los } \\
\text { hashtags } \\
\text { únicos }\end{array}$ & $\begin{array}{c}\text { Hashtags } \\
\text { propios entre } \\
\text { los TT }\end{array}$ & $\begin{array}{c}\text { Inserción en } \\
\text { agenda de } \\
\text { TT }\end{array}$ \\
\hline Barberá & 272 & 26 & 10.46 & 4 & 4 & $3.92 \%$ \\
Ribó & 67 & 21 & 3.19 & 5 & 2 & $4.90 \%$ \\
Giner & 2 & 2 & 1.00 & 1 & 1 & $0.98 \%$ \\
Calabuig & 65 & 36 & 1.81 & 4 & 4 & $3.92 \%$ \\
Peris & 433 & 64 & 6.77 & 18 & 4 & $17.64 \%$ \\
\hline
\end{tabular}

Así, el partido que en más ocasiones logró que sus hashtags alcanzaran la condición de trending topic fue el PSPV-PSOE (7), seguido de Ciudadanos (6), Compromís (4) y, por último, de València en Comú (3). A su vez, los candidatos que en mayor medida lo hicieron fueron Peris, Barberá y Calabuig (4), Ribó (2) y, por último, Giner (1).

Sin embargo, la influencia en el establecimiento de la agenda de tendencias que hemos señalado hasta ahora es meramente cuantitativa, de manera que los resultados del análisis son diferentes al computar la duración acumulada como tendencia por los hashtags propios que en algún momento recibieron la consideración de trending topics, como se muestra en la Tabla 13.

De esta forma, se constata que la formación cuyos hashtags estuvieron alineados durante más tiempo con la agenda política de la comunidad de usuarios de Twitter en el área de Valencia fue València en Comú (32 horas), seguida del PSPV-PSOE (30 horas), PPCV (26 horas y 40 minutos), Compromís (21 horas y 50 minutos) y, por último, Ciudadanos (20 horas y 55 minutos). Pese a ello, los hashtags convertidos en tendencia no fueron iniciados en su mayoría por las cuentas de los partidos y candidatos en el ámbito municipal, sino que en ocasiones fueron iniciados por las cuentas de los ámbitos autonómico y nacional. Destaca, en este sentido, que todos los hahstags propios que alcanzaron la condición de trending topic durante más de cinco horas (\#votapp, \#sumacompromis, \#yovotociudadanos, \#votapsoe, \#nosvemosenlaplaza, \#hagamoshistoria24m, \#haciael24m y \#quieroapodemosenelgobierno) estuvieran iniciados por cuentas corporativas de diferente ámbito al municipal. 
Tabla 13. Duración como trending topics en el área de Valencia de los hashtags promovidos por partidos y candidatos

\begin{tabular}{|c|c|c|c|}
\hline Cuenta & Hahstag propio convertido en TT & $\begin{array}{r}\text { Duración in- } \\
\text { dividual }\end{array}$ & $\begin{array}{l}\text { Duración } \\
\text { agregada }\end{array}$ \\
\hline \multirow[t]{4}{*}{ Barberá } & \# ritaalcaldesa & oh $35^{\prime}$ & $26 \mathrm{~h} 4 \mathrm{O}^{\prime}$ \\
\hline & \#ahorappcv & Sin datos & \\
\hline & \#votapp & $25{\mathrm{~h} 2 \mathrm{O}^{\prime}}^{\prime}$ & \\
\hline & \#votappcv & oh $45^{\prime}$ & \\
\hline \multirow{4}{*}{$\begin{array}{l}\text { Compromís y } \\
\text { Ribó }\end{array}$} & \# ritaleaks & $4 \mathrm{~h} 45^{\prime}$ & $21 \mathrm{~h} 5 \mathrm{o}^{\prime}$ \\
\hline & \#sumacompromís & $8 \mathrm{~h} \mathrm{oo'}$ & \\
\hline & \#monicapresidenta & $4 \mathrm{~h} 55^{\prime}$ & \\
\hline & \#valentuits & $4 \mathrm{~h} 10^{\prime}$ & \\
\hline \multirow{6}{*}{$\begin{array}{l}\text { Ciudadanos y } \\
\text { Giner }\end{array}$} & \#valencianaranja & Sin datos & $20 h 55^{\prime}$ \\
\hline & \#yovotociudadanos & 13 h oo' & \\
\hline & \#ilusionnaranja & $4 \mathrm{~h} 25^{\prime}$ & \\
\hline & \# españapidecambio & Sin datos & \\
\hline & \# españaciudadana & $2 \mathrm{~h} 55^{\prime}$ & \\
\hline & \#apoderadoscs & oh $35^{\prime}$ & \\
\hline \multirow{8}{*}{$\begin{array}{l}\text { PSPV y Cala- } \\
\text { buig }\end{array}$} & \#alcaldecalabuig & $1 \mathrm{~h} 15^{\prime}$ & $30 \mathrm{oh}$ oo' \\
\hline & \#votapsoe & 9h oo' & \\
\hline & \#lovamosaarreglar & $2 \mathrm{~h} 10^{\prime}$ & \\
\hline & \#ximopresident & $2 \mathrm{~h} 15^{\prime}$ & \\
\hline & \#digosivotopsoe & $4 \mathrm{~h} 40^{\prime}$ & \\
\hline & \#nosvemosenlaplaza & $11 \mathrm{~h} \mathrm{oo'}$ & \\
\hline & \#vlcnews2av & Sin datos & \\
\hline & \#vlcnews2gv & Sin datos & \\
\hline \multirow[t]{5}{*}{ VALC y Peris } & \#hagamoshistoria24m & $8 \mathrm{~h} \mathrm{oo'}$ & $32 \mathrm{~h} \mathrm{oo'}$ \\
\hline & \#haciael24m & $10 \mathrm{~h} \mathrm{o5}$ & \\
\hline & \#apoderaelcambio & Sin datos & \\
\hline & \#quieroapodemosenelgobierno & 9h oo' & \\
\hline & \#primaveradelcambio & $4 \mathrm{~h} 55^{\prime}$ & \\
\hline
\end{tabular}




\section{Las agendas temáticas de los cibermedios}

Por último, en referencia a la PI4, que planteaba ċcuál es la composición de las agendas temáticas de los cibermedios y en qué medida están alineadas con las agendas partidistas?, los seis periódicos digitales analizados confirieron a la política sectorial un espacio principal en sus agendas, al igual que ocurrió con la mayoría de las webs de campaña y a diferencia de lo detectado en las agendas que partidos y candidatos presentaron en sus cuentas de Twitter. Sin embargo, como muestra la Tabla 14, el peso de los policy issues en las diferentes agendas mediáticas fue desigual. Mientras en los cibermedios vinculados a diarios locales con edición impresa, como Levante-EMV y Las Provincias, la presencia de dicho bloque superó ampliamente al segundo y se situó en torno a la mitad de la agenda, en el resto de los medios su preponderancia no fue tan evidente. A diferencia de los demás, los dos medios mencionados no se limitaron a cubrir la campaña o los casos de corrupción, sino que continuaron con la cobertura que sus secciones de información municipal realizaban habitualmente de la gestión del Ayuntamiento de Valencia y de la actualidad no partidista ni institucional relacionada con la ciudad.

Tabla 14. Composición de las agendas de los cibermedios por bloques temáticos

\begin{tabular}{lcccccc}
\hline Bloque & El País & El Mundo & Levante & LP & V. Plaza & El Diario \\
\hline Political issues & $32.98 \%$ & $32.75 \%$ & $17.69 \%$ & $27.57 \%$ & $32.09 \%$ & $30.60 \%$ \\
Policy issues & $35.11 \%$ & $36.26 \%$ & $55.58 \%$ & $46.30 \%$ & $33.95 \%$ & $41.04 \%$ \\
$\begin{array}{l}\text { Campaign } \\
\text { issues }\end{array}$ & $18.09 \%$ & $23.39 \%$ & $22.50 \%$ & $22.63 \%$ & $28.84 \%$ & $20.15 \%$ \\
Personal issues & $13.83 \%$ & $7.60 \%$ & $4.23 \%$ & $3.50 \%$ & $5.12 \%$ & $8.21 \%$ \\
\hline
\end{tabular}

La distribución por áreas del bloque de policy issues presentó una fractura similar como consecuencia de idénticos motivos. Como se observa en la Tabla 15, todos los cibermedios analizados priorizaron de forma clara los asuntos relacionados con el buen gobierno, sobre todo a partir de informaciones sobre corrupción y uso partidista de fondos públicos. Sin embargo, el peso de dicha área en las agendas de Levante-EMV y de Las Provincias fue menor que el que ocupa en el resto de los medios digitales analizados. 
Tabla 15. Composición de las agendas de los cibermedios por áreas sectoriales

\begin{tabular}{|c|c|c|c|c|c|c|}
\hline Policy issues & El País & El Mundo & Levante & LP & V. Plaza & El Diario \\
\hline Hacienda e inversiones & $O \%$ & $0.58 \%$ & $1.54 \%$ & $1.23 \%$ & $1.40 \%$ & $0.75 \%$ \\
\hline Buen gobierno & $24.47 \%$ & $28.65 \%$ & $14.23 \%$ & $11.11 \%$ & $19.53 \%$ & $26.87 \%$ \\
\hline Economía y empleo & o\% & $1.75 \%$ & $6.15 \%$ & $4.53 \%$ & $2.79 \%$ & $2.99 \%$ \\
\hline $\begin{array}{l}\text { Urbanismo y espacios natura- } \\
\text { les }\end{array}$ & $4.26 \%$ & $1.17 \%$ & $6.54 \%$ & $6.38 \%$ & $1.86 \%$ & $5 \cdot 97 \%$ \\
\hline $\begin{array}{l}\text { Mov., esp. público y c. am- } \\
\text { biental }\end{array}$ & o\% & $1.17 \%$ & $9.62 \%$ & $8.02 \%$ & $0.47 \%$ & $1.49 \%$ \\
\hline Seguridad ciudadana & o\% & $0.58 \%$ & $2.12 \%$ & $0.82 \%$ & $0.47 \%$ & o\% \\
\hline Política social & $3.19 \%$ & $0.58 \%$ & $5 \cdot 38 \%$ & $2.47 \%$ & $1.40 \%$ & $1.49 \%$ \\
\hline Educación, cultura y deporte & $1.06 \%$ & $1.75 \%$ & $8.85 \%$ & $10.29 \%$ & $4.65 \%$ & $1.49 \%$ \\
\hline Otros & $2.13 \%$ & o\% & $1.15 \%$ & $1.44 \%$ & $1.40 \%$ & o\% \\
\hline
\end{tabular}

Así, mientras el resto de áreas ocupó espacios residuales en El País, El Mundo, El Diario y Valencia Plaza, las agendas temáticas de Levante-EMV y de Las Provincias otorgaron un lugar secundario, pero no marginal a los asuntos relacionados con las áreas de urbanismo y espacios naturales, movilidad y espacio público, educación, patrimonio y cultura festiva, y economía y empleo. Los temas relacionados con las áreas de hacienda municipal y de seguridad ciudadana ocuparon, en todos los casos, lugares residuales. De tal forma, aunque no resulte posible establecer una equivalencia clara entre las agendas mediáticas y las partidistas oficiales, sí es evidente que la primacía del área de buen gobierno en todos los cibermedios fue concordante con los intereses evidenciados por Compromís y el PSPV-PSOE en la composición de sus agendas.

\section{Discusión y conclusiones}

Los partidos priorizaron en sus agendas temáticas oficiales los policy issues y no los temas relacionados con el transcurso de la propia campaña, en especial aquellos con presencia en la corporación municipal durante el mandato 2011-2015. La composición del primer nivel de la agenda temática partidista oficial respondió así durante la campaña electoral al trabajo sectorial previo desarrollado por las formaciones, más intenso por motivos obvios en el caso de los partidos consolidados que en el de los emergentes. Las formaciones destacaron las áreas de gestión más próximas a sus discursos tradicionales: el PPCV se centró en la recuperación económica, la creación de empleo y las inversiones realizadas durante su mandato; el centroizquierda consolidado de Compromís y el PSPV-PSOE, en la transparencia, la participación ciudadana, el uso de los fondos públicos, el despilfarro y la corrupción; la izquierda emergente de València en Comú, en el empleo y las políticas sociales. Además, tanto el 
partido en el gobierno como los dos emergentes centraron sus mensajes sobre política general en el mismo eje con diferentes interpretaciones en función de sus intereses electorales: el Partido Popular, estabilidad-incertidumbre; Ciudadanos, continuidad-cambio; y València en Comú, vieja-nueva política. Así, la primacía de unas áreas de gestión sobre otras respondió sobre todo a la estrategia, la ideología y la trayectoria de cada formación. La composición de las agendas en estos comicios fue congruente con las transmitidas por las mismas formaciones en las Elecciones al Parlamento Europeo de 2014 (López et al., 2015) y confirmó, además, la adecuación de los temas difundidos por los partidos a lo que sus seguidores esperaban de ellos (López-López et al., 2020), lo cual reforzó el establecimiento de un mensaje cognitivamente congruente con las expectativas de sus comunidades, aspecto especialmente relevante en los procesos de fusión de agendas.

Sin embargo, dichas agendas no se reflejaron por completo en los mensajes emitidos por partidos y candidatos en Twitter, que orientaron de manera generalizada su actividad en la red de microblogging a la difusión de sus actividades de campaña y al llamamiento al voto. Ello, unido a la escasa interacción con los seguidores en dichos comicios (Gamir Ríos, 2020), corrobora la unidireccionalidad en el uso de esta red por parte de los partidos (Giansante, 2015), así como su utilización para la movilización de simpatizantes (Kreiss, 2016). Además, las formaciones no se limitaron a adaptar sus agendas en función de las propiedades y posibilidades de la red de microblogging con la inversión de la presencia otorgada a los bloques de policy issues y de campaign issues, sino que construyeron agendas diferenciadas a través de sus candidatos, adaptadas a dichas posibilidades pero que, al mismo tiempo, redefinieron la jerarquía de prioridades sectoriales respecto a las agendas oficiales. Los alcaldables aprovecharon así sus cuentas para complementar las agendas oficiales.

Por su parte, la comunidad de usuarios de Twitter del área de Valencia prestó una atención menor a los asuntos políticos y, cuando lo hizo, fue a propósito de aspectos relacionados con la campaña, como actos públicos, llamamientos al voto o anécdotas, y no con aspectos sectoriales concretos. La influencia de los partidos en la conversión en tendencia de las etiquetas que promovieron fue, en todos los casos, escasa. Ello, sin embargo, no apunta necesariamente a una disonancia cognitiva entre los mensajes partidistas y la percepción de la realidad de sus seguidores, aspecto que hubiera aumentado la latencia en la propagación de mensajes (Calvo \& Aruguete, 2018; López-López et al., 2020), sino que es atribuible al escaso interés que la política municipal suscitaba entre los usuarios de la red. De hecho, los hashtags partidistas que durante más tiempo ostentaron dicha consideración estuvieron promovidos, además, desde el ámbito nacional, lo que evidencia también un alto grado de seguidismo de la agenda tuitera española por parte de la comunidad local de usuarios.

Los periódicos digitales centraron sus agendas en el área de buen gobierno del bloque de política sectorial, en concreto en los asuntos relacionados con la corrupción y el uso partidista de fondos públicos, y en los actores partidistas relacionados con el Partido Popular, sobre todo Rita Barberá, que en todos los casos excepto en el de Las Provincias fueron objeto de un tratamiento informativo negativo. La única diferencia notable entre las composiciones de las diferentes agendas mediáticas 
radica en la mayor cobertura que dicho cibermedio y Levante-EMV realizaron de la actividad habitual de gestión municipal, atribuible al ámbito de difusión de sus referentes impresos, exclusivamente provincial. Aunque no existieron procesos absolutos de asimilación de las agendas partidistas por parte de los cibermedios, sus temáticas sí estuvieron parcialmente alineadas con los intereses estratégicos de las formaciones que no ostentaban el gobierno. Sin embargo, y pese a que uno de los ejemplos de supuesto uso partidista de fondos públicos (\#Ritaleaks) fue difundido desde el principio por Compromís, no estimamos que este parcial alineamiento fuera consecuencia de la modulación del contexto periodístico por parte de los tuits políticos (Parmelee, 2013), pues muchos de dichos temas llevaban meses instalados en la agenda mediática.

Aunque consideramos que los resultados del trabajo resultan relevantes en el estudio de la transmisión de las agendas políticas en Twitter y en los cibermedios durante una campaña electoral, y pese a que las Elecciones Locales de 2015 en la ciudad de Valencia ejemplificaron la situación electoral española, sería conveniente responder a las mismas preguntas en investigaciones futuras tomando como objeto de estudio una campaña de ámbito nacional. Del mismo modo, dada la relevancia de la política municipal desarrollada en las capitales autonómicas en la construcción de las agendas públicas locales, resultaría de interés la realización de un estudio comparativo de los mismos aspectos en las campañas desarrolladas en diversas ciudades durante unos mismos comicios.

\section{Declaración de conflicto de intereses}

El autor no informó ningún posible conflicto de intereses.

\section{Referencias}

Alonso-Muñoz, L., Miquel-Segarra, S., \& Casero-Ripollés, A. (2016). Un potencial comunicativo desaprovechado. Twitter como mecanismo generador de diálogo en campaña electoral. Obra Digital, (11), 39-59. https://doi.org/10.25029/od.2016.100.11

Alonso-Muñoz, L., \& Casero-Ripollés, A. (2018). Political agenda on Twitter during the 2016 Spanish elections: issues, strategies, and users' responses. Communication \& society, 3(3), 7-23.

Aruguete, N. (2017). Agenda building. Revisión de la literatura sobre el proceso de construcción de la agenda mediática. Signo y Pensamiento, 36(70), 36-52. https://doi.org/10.11144/Javeriana.syp36-70.abrl

Baviera, T. (2018). Influence in the political Twitter sphere: Authority and retransmission in the 2015 and 2016 Spanish General Elections. European Journal of Communication, 33(3), 321-337. https://doi.org/10.1177/0267323118763910

Bardin, L. (1986). Análisis de contenido. Akal.

Boix Palop, A., \& López García, G. (2014). El significado de las Elecciones Europeas de 2014 en España: giro a la izquierda y hundimiento del bipartidismo. Unión Europea Aranzadi, 7, 69-93.

Campos-Domínguez, E. (2017). Twitter y la comunicación política. El Profesional de la Información, 26(5), 785-793. https://doi.org/10.3145/epi.2017.sep.01 
Calvo, E., \& Aruguete, A. (2018). \#Tarifazo. Medios tradicionales y fusión de agenda en redes sociales. InMediaciones de la Comunicación, 13(1), 189-213. https://doi.org/10.18861/ic.2018.13.1.2831

Casero Ripollés, A. (2009). El control político de la información periodística. Revista Latina de Comunicación Social, (64), 354-366. https://doi.org/10.4185/RLCS-64-2009-828-354-366

Casero-Ripollés, A., Feenstra, R. A., \& Tormey, S. (2016). Old and new media logics in an electoral campaign: The case of Podemos and the two-way street mediatization of politics. The International Journal of Press/Politics, 21(3), 378-397. https://doi.org/10.1177/1940161216645340

Casero-Ripolles, A., Miquel-Segarra, S., \& Alonso-Muñoz, L. (2016). The dialogic potential of Twitter in electoral campaign. The case of PSOE and Podemos in Spain [ponencia]. 11th Iberian Conference on Information Systems and Technologies (CISTI), Las Palmas, España. https://doi.org/10.1109/cisti.2016.7521592

Castells, M. (2009). Comunicación y poder. Alianza Editorial.

Cervi, L., \& Roca, N. (2017). La modernización de la campaña electoral para las elecciones generales de España en 2015. ¿Hacia la americanización? Comunicación y Hombre, (13), 133-150. https://doi.org/10.32466/eufv-cyh.2017.13.219.133-150

Cervi, L., \& Roca Trenchs, N. (2018). El uso de Twitter por parte de los principales candidatos en las campañas electorales para las elecciones generales españolas: 2011 y 2015. ¿Brecha digital y generacional? Doxa Comunicación, (26), 99-126. https://doi.org/10.31921/doxacom.n26a5

Chandwick, A. (2013). The Hybrid Media System. Politics and Power. Oxford University Press.

Dader, J. L. (2017). Campañas políticas “online”: La realidad española frente al horizonte internacional del "tecnocabildeo". En J. L. Dader \& E. Campos (coords.), La búsqueda digital del voto. Cibercampañas Electorales en España 2015-16 (pp. 75-140). Tirant lo Blanch.

Fenoll, V., \& Cano-Orón, L. (2019). Communicative differences between emergent and traditional parties: An analysis of Facebook posts from Spain's 2015 general election campaign. Zer, 24(46), 37-51. https://doi.org/10.1387/zer.20225.

Gamir Ríos, J. (2016). Blogs, Facebook y Twitter en las Elecciones Generales de 2011. Estudio cuantitativo del uso de la web 2.0 por parte de los cabezas de lista del PP y del PSOE. Dígitos, Revista de Comunicación Digital, (2), 101-120.

Gamir Ríos, J. (2020). El uso político de Twitter en la campaña de las Elecciones Locales de 2015 en la ciudad de València. Miguel Hernández Communication Journal, (11), 35-54.

Gamir Ríos, J., Cano Orón, L., \& Calvo, D. (2017). La campaña electoral de 2015 en cifras. La presencia en la blogosfera, Facebook y Twitter de los cabezas de lista provinciales de PP, PSOE, Podemos y Ciudadanos. En G. López García \& L. Valera Ordaz (Eds.), Pantallas electorales. El discurso de partidos, medios y ciudadanos en la campaña de 2015 (pp. 41-58). Editorial UOC.

García-Ortega, C., \& Zugasti Azagra, R. (2018). Gestión de la campaña de las elecciones generales de 2016 en las cuentas de Twitter de los candidatos: entre la autorreferencialidad y la hibridación mediática. El Profesional de la Información, 27(6), 1.215-1.225. https://doi.org/10.3145/epi.2018.nov.05

Giansante, G. (2015). La comunicación política online. Editorial UOC.

Igartua, J. J. (2006). Métodos cuantitativos de investigación en comunicación. Bosch.

Jenkins, H. (2008). Convergence Culture: La cultura de la convergencia de los medios de comunicación. Paidós. 
Jungherr, A. (2014). The logic of political coverage on Twitter: Temporal dynamics and content. Journal of Communication, 64(2), 239-259. https://doi.org/10.1111/jcom.12087

Jungherr, A. (2015). Twitter use in election campaigns: A systematic literature review. Journal of $\begin{array}{llll}\text { Information } \quad \text { Technology } \quad \text { Politics, } & \text { 13(1), }\end{array}$ https://doi.org/10.1080/19331681.2015.1132401

Kreiss, D. (2016). Seizing the moment: The presidential campaigns' use of Twitter during the 2012 electoral cycle. New Media \& Society, 18(8), 1473-1490. https://doi.org/10.1177/1461444814562445

Krippendorff, K. (1990). Metodología de análisis de contenido. Paidós

Lasorsa, D. L., Lewis, S. C., \& Holton, A. E. (2012). Normalizing Twitter: Journalism practice in an emerging communication space. Journalism Studies, 13(1), 19-36. https://doi.org/10.1080/1461670x.2011.571825

López-García, G. (2016). 'Nuevos’ y ‘viejos' liderazgos: la campaña de las elecciones generales españolas de 2015 en Twitter. Communication \& Society, 29(3), 149-167. https://doi.org/10.15581/o03.29.3.149-168

López García, G., Gámir Ríos, J., García Ull, F. J., Llorca Abad, G., Cano Orón, L., \& González Esteban, J. L. (2015). El debate sobre Europa en Twitter. Discursos y estrategias de los candidatos de las elecciones al Parlamento Europeo de 2014 en España. Revista de Estudios Políticos, (170), 213-246. https://doi.org/10.18042/cepc/rep.170.07

López-López, P. C., Castro Martínez, P., \& Oñate, P. (2020). Agenda melding y teorías de la comunicación: la construcción de la imagen de los actores políticos en las redes sociales. Cuadernos del Centro de Estudios en Diseño y Comunicación, (112), 21-34.

López-Meri, A., Marcos-García, S., \& Casero-Ripollés, A. (2017). What do politicians do on Twitter? Functions and communication strategies in the Spanish electoral campaign of 2016. El Profesional de la Información, 26(5), 795-804. https://doi.org/10.3145/epi.2017.sep.02

Machado Flores, N., \& Capdevilla Gómez, A. (2016). Interacción y debate en Twitter en las elecciones españolas de mayo de 2015: ¿promesa tecnológica o realidad virtual? Obra digital, (11), 61-83. https://doi.org/10.25029/od.2016.96.11

Mazzoleni, G. (2010). La comunicación política. Alianza Editorial.

Nitschke, P., Donges, P., \& Schade, H. (2014). Political organizations' use of websites and Facebook. New Media \& Society, 18(5), 744-764. https://10.1177/1461444814546451

Parmelee, J. H. (2013). The agenda building function of political tweets. New Media \& Society, 16(3), 434-45o. https://doi.org/10.1177/1461444813487955

Shaw, D. L, McCombs, M., Weaver, D. H., \& Hamm, B. J. (1999). Individuals, groups, and agenda melding: a theory of social dissonance. International Journal of Public Opinion Research, 11(1), 2-24. https://doi.org/10.1093/ijpor/11.1.2

Vaccari, C., \& Valeriani, A. (2016). Party Campaigners or Citizen Campaigners? How Social Media Deepen and Broaden Party-Related Engagement. The International Journal of Press/Politics, 21(3), 294-312. https://doi.org/10.1177/1940161216642152

Vargo, C. J., Guo, L., McCombs, M., \& Shaw, D. L. (2014). Network issue agendas on Twitter during the 2012 U.S. presidential election. Journal of Communication, 64(2), 296-316. https://doi.org/10.1111/jcom.12089

Vergeer, M. (2015). Twitter and political campaigning. Sociology Compass, 9(9), 745-760. https://doi.org/10.1111/soc4.12294 